\title{
Multiobjective optimization for nuclear fleet evolution scenarios using COSI
}

\author{
David Freynet $^{1 *}$, Christine Coquelet-Pascal ${ }^{1}$, Romain Eschbach ${ }^{1}$, Guillaume Krivtchik ${ }^{1}$, and Elsa Merle-Lucotte ${ }^{2}$ \\ ${ }^{1}$ CEA, DEN, Cadarache, DER, SPRC, LECy, 13108 Saint-Paul-lez-Durance, France \\ 2 LPSC-IN2P3-CNRS, UJF, Grenoble INP, 53 rue des Martyrs, 38026 Grenoble, France
}

Received: 5 October 2015 / Accepted: 17 December 2015

Published online: 4 March 2016

\begin{abstract}
The consequences of various fleet evolution options on material inventories and flux in fuel cycle and waste can be analysed by means of transition scenario studies. The COSI code is currently simulating chronologically scenarios whose parameters are fully defined by the user and is coupled with the CESAR depletion code. As the interactions among reactors and fuel cycle facilities can be complex, and the ways in which they may be configured are many, the development of optimization methodology could improve scenario studies. The optimization problem definition needs to list: (i) criteria (e.g. saving natural resources and minimizing waste production); (ii) variables (scenario parameters) related to reprocessing, reactor operation, installed power distribution, etc.; (iii) constraints making scenarios industrially feasible. The large number of scenario calculations needed to solve an optimization problem can be time-consuming and hardly achievable; therefore, it requires the shortening of the COSI computation time. Given that CESAR depletion calculations represent about 95\% of this computation time, CESAR surrogate models have been developed and coupled with COSI. Different regression models are compared to estimate CESAR outputs: first- and second-order polynomial regressions, Gaussian process and artificial neural network. This paper is about a first optimization study of a transition scenario from the current French nuclear fleet to a Sodium Fast Reactors fleet as defined in the frame of the 2006 French Act for waste management. The present article deals with obtaining the optimal scenarios and validating the methodology implemented, i.e. the coupling between the simulation software COSI, depletion surrogate models and a genetic algorithm optimization method.
\end{abstract}

\section{Introduction}

\subsection{Transition scenario studies}

Nuclear systems composed of reactors with varied fuels and cycle facilities (enrichment, fabrication and reprocessing plants, interim and waste storages) are complex and in constant evolution. Transition scenario studies assist decision makers in listing the strengths and weaknesses of different strategies for a nuclear fleet evolution. These studies involve the tracking of the batches of materials and the evaluation of their depletion in the fuel cycle over a defined period.

COSI is a code developed by the CEA's Nuclear Energy Division and used to simulate the evolution of a nuclear reactor fleet and the associated fuel cycle facilities [1]. COSI takes as input parameters fuel cycle facilities and reactors features, fuel types characteristics and succession of

\footnotetext{
* e-mail: david.freynet@cea.fr.
}

loadings. Front-end, back-end and waste paths define relations between these facilities as shown in Figure 1. It should be noted that reactors are defined by commissioning and shutdown dates, and reprocessing plants are defined by these dates, reprocessing capacities and strategy features. COSI provides outputs about the isotopic masses in the fuel cycle facilities and reactors over a defined period. Post processing calculations give access to physical quantities of interest: activity, radiotoxicity, decay heat, etc.

COSI is coupled with the CESAR depletion code, developed by the CEA's Nuclear Energy Division and AREVA, which performs every depletion (irradiation and cooling) calculation during the scenario simulation [2]. CESAR is the reference code used at La Hague reprocessing plant. Using CESAR requires one-group cross-sections libraries linked to fuel types loaded in the reactors. The production of these libraries requires neutronic calculations (APOLLO and ERANOS) and is separated from the depletion calculations. COSI coupled with the CESAR5.3 version is tracking 109 heavy nuclides $(\mathrm{Tl} \rightarrow \mathrm{Cf})$ and 212 fission products $(\mathrm{Zn} \rightarrow \mathrm{Ho})$. 


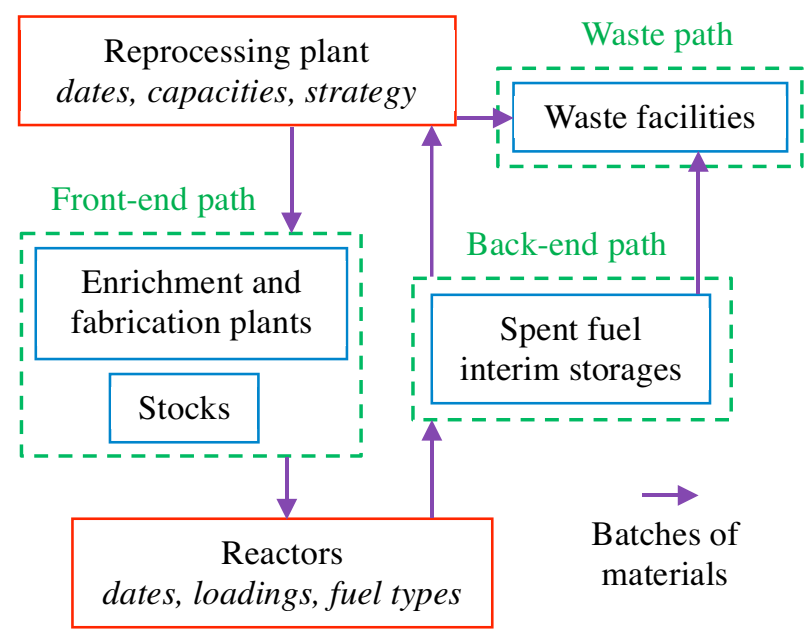

Fig. 1. COSI simplified data set operating diagram.

\subsection{Multiobjective optimization}

COSI is currently simulating chronologically scenarios whose parameters are fully defined by the user. The aim of this paper is to define a methodology for the automatic search of scenarios which are adapted to a strategic problem. Indeed the future French nuclear fleet should meet numerous and often conflicting criteria for different stakeholders such as saving natural resources and minimizing nuclear waste production. Such criteria have to be minimized or maximized according to some scenario parameters (COSI inputs).

Solving an optimization problem requires a large number of scenario calculations, which could be timeconsuming and hardly achievable. Indeed this time can vary from a few minutes to a few hours according to the scenario assumptions and the number of isotopes tracked. Because CESAR calculations represent approximately $95 \%$ of the COSI computation time, depletion simplified models have been introduced to shorten depletion calculations during the scenario computation. Consequently, CESAR-based irradiation surrogate models are developed using the sensitivity and uncertainty platform URANIE developed by the CEA's Nuclear Energy Division [3].

Because of the large numbers of scenario parameters and criteria available to define an optimization problem, we opt to use metaheuristics as optimization methods. The URANIE's genetic algorithm (GA) is considered for the present optimization studies. Therefore, URANIE is used both for the surrogate models development and the optimization studies.

The methodology for performing multiobjective optimization using COSI is represented in Figure 2.

The development of CESAR surrogate models is discussed in Section 2. Then the COSI sped up version using these simplified models is validated by comparing its results to COSI, this study is also presented in Section 2. Finally, an application of this methodology for the optimization of a transition scenario from the current Pressurized Water Reactors (PWR) French nuclear fleet to a fleet of Sodium Fast Reactors (SFR) is presented in Section 3 .

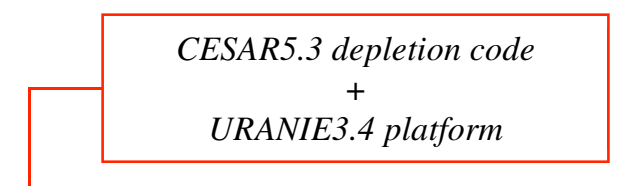

Part 2: development of CESAR surrogate models

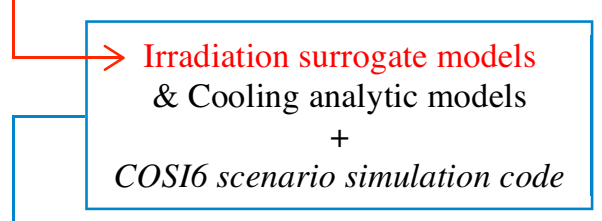

Part 2.4: validation of COSI sped up version

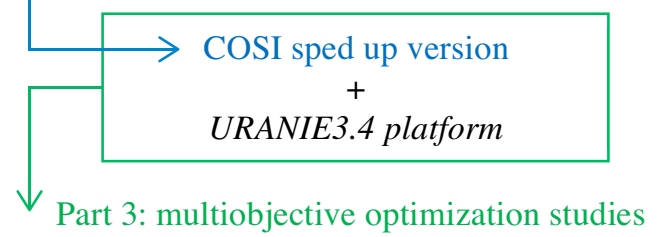

Fig. 2. Global multiobjective optimization methodology.

Other works address similar optimization problems using different simulation software such as VISION and CAFCA codes $[4,5]$.

\section{Irradiation surrogate models}

\subsection{Methodology}

As seen previously, multiobjective optimization studies require the shortening of the COSI computation time and so the CESAR one. A way to gain time at cost to a satisfactory estimation error is developing CESAR surrogate models. These models can replace CESAR for irradiation calculations throughout the COSI computation and so have the same inputs and outputs as CESAR.

CESAR input parameters define the fuel assembly composition and irradiation features:

- the fresh fuel assembly isotopic composition defines the isotope (denoted $i$ ) mass fractions in the fuel noted $y_{i}=m_{i} / m_{\text {fuel }}\left(\sum_{i} y_{i}=1\right)$;

- the burnup to achieve noted BU in MWd/tHM;

- the irradiation time noted $\Delta t$ in days.

Thereafter, let $x=\left\{\forall i y_{i} ; B U ; \Delta t\right\}$ be the $N$-terms vector of CESAR input parameters.

CESAR outputs are the results of depletion calculation, i.e. the spent fuel isotopic composition. These outputs are calculated as final concentrations noted $\mathrm{C}_{j}(x)$ where $j$ denotes spent fuel isotopes in atoms/ton.

The development of irradiation surrogate models (see Fig. 3) consists first in defining designs of experiments of the CESAR input parameters and associated outputs. These designs are defined using Latin hypercube sampling method (LHS) because of its high space-filling performance. The number of $x$ vectors defined for each design is set to 500 . Then a regression model is applied to produce a surrogate model. Surrogate models are noted $C_{j}$ as the functions 


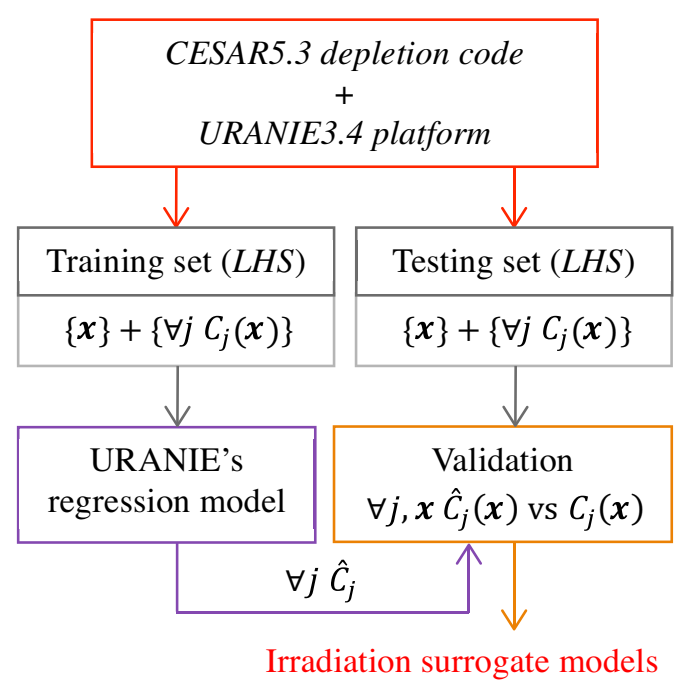

Fig. 3. Surrogate models development methodology.

estimating the $\mathrm{C}_{j}$ CESAR results. Finally, quality indicators are performed on each surrogate model to ensure that the prediction power is satisfactory.

We make one surrogate model per tracked isotope per fuel type considered in the application scenario. For each fuel type, we make two designs of CESAR calculations: one for the regression step (named the training set) and another one for the validation step (named the testing set). All these operations are carried out with the URANIE platform.

The use of CESAR surrogate models coupled with the COSI code has already been introduced for uncertainty propagation studies in nuclear transition scenarios $[6,7]$.

\subsection{Regression models}

CESAR surrogate models are developed using a regression method on the training set. The following methods are compared:

- first- (LR) and second-order (PR) polynomial regressions;

- Gaussian process (GP);

- artificial neural network (ANN).

Polynomial regression is a well-known approach to adjust a set of points by a function. Applied to CESAR calculations training set, the estimator is defined by equation (1) (LR) or equation (2) (PR):

$$
\begin{gathered}
\forall x \hat{C}_{j}(x)=\alpha_{0}+\sum_{n=1}^{N} \alpha_{n} x_{n} \\
\forall x C_{j}(x)=\alpha_{0}+\sum_{n=1}^{N} \alpha_{n} x_{n}+\sum_{p=1}^{N} \sum_{q=1}^{N} \alpha_{p q} x_{p} x_{q} .
\end{gathered}
$$

Polynomial regression consists in finding the $\alpha$ parameters giving the best model adjustment on the training set. CESAR surrogate models development with polynomial regression is detailed in a past work [6].
Gaussian process is a non-parametric regression method using a deterministic function and a correlation function involving parameters determined by maximum-likelihood estimation [8].

Artificial neural network is used in its single-layer perceptron form, i.e. there are no cycles and loops in the network and only one output neuron. Applied to CESAR calculations training set, the estimator is defined as:

$$
\forall x \hat{C}_{j}(x)=\alpha_{0}+\sum_{h=1}^{H} \alpha_{h} S\left(\alpha_{0 h}+\sum_{n=1}^{N} \alpha_{n h} x_{n}\right)
$$

where $S(x)=1 /(1+\exp (-x))$ is the sigmoid function and $h$ denotes the hidden neuron. A backpropagation algorithm is applied to calculate the $\alpha$ weights by minimizing the estimation root mean square error. CESAR surrogate models development with ANN is also presented in another work [7].

\subsection{Validation results}

Surrogate models have been defined according to their use in optimization studies. Indeed the set of scenarios considered in this paper is extracted from the 2006 French Act for waste management which involves estimating PWR UOX, PWR MOX, PWR ERU and SFR MOX fuel types depletion. The validation step has to be applied to all of the surrogate models. Only the results of the $\hat{C}_{P u 239}$ and $\hat{C}_{C m 244}$ estimators for a PWR MOX irradiation are presented here, because of the importance of their accurate estimation and their non-linear evolution. Results shown in this part consider that GP deterministic function is linear, GP correlation function is Matérn 3/2 and the ANN number of hidden layers is 6 .

Validating surrogate model rests upon the evaluation of indicators quantifying the quality of the regression and above all the estimator capacity to reckon the CESAR outputs. These indicators have to be representative of different estimation errors and are calculated using the testing set. Generally the predictivity coefficient $q^{2}$ acts as the main indicator for validating surrogate models [8]. Yet irradiation surrogate models are coupled with COSI which is repeatedly run during the optimization process. Thus, estimation error needs to be known to check that its impact is negligible on COSI outputs. For each testing $x$ vector and surrogate model, let $\Delta_{j}(x)$ be the absolute estimation error divided by the mean of $C_{j}(x)$ on the testing vectors:

$$
\Delta_{j}(x)=\left|\hat{C}_{j}(x)-C_{j}(x)\right| / \bar{C}_{j}
$$

Calculating the mean and maximal values of this indicator on the testing set enables estimating the surrogate model quality. Replacing the denominator of equation (4) by $C_{j}(x)$, i.e. calculating the relative error, leads to high errors for low values of output concentrations. These cases are not significant for scenario studies because they are unnecessary to get a good estimation of the spent fuel 
Table 1. Indicators of validation for PWR MOX ${ }^{239} \mathrm{Pu}$ and ${ }^{244} \mathrm{Cm}$ concentration estimations by surrogate models.

\begin{tabular}{|c|c|c|c|c|}
\hline \multirow{2}{*}{$\begin{array}{l}\text { Regression } \\
\text { method }\end{array}$} & \multicolumn{2}{|c|}{$j={ }^{239} \mathrm{Pu}$} & \multicolumn{2}{|c|}{$j={ }^{244} \mathrm{Cm}$} \\
\hline & $\operatorname{Mean}_{x} \Delta_{j}(\%)$ & $\operatorname{Max}_{x} \Delta_{j}(\%)$ & $\operatorname{Mean}_{x} \Delta_{j}(\%)$ & $\operatorname{Max}_{x} \Delta_{j}(\%)$ \\
\hline LR & 1.3 & 6.3 & 4.4 & 20 \\
\hline PR & 0.093 & 0.69 & 0.71 & 3.1 \\
\hline GP & 0.22 & 2.5 & 0.85 & 5.6 \\
\hline
\end{tabular}

composition. Consequently, the definition given here is preferred. Error results are shown in Table 1.

This comparison study implies to consider ANN for all the CESAR surrogate models development.

\subsection{Toward a COSI sped up version}

Cooling calculation can be sped up using cooling surrogate models, but the analytic solutions of the Bateman equation with no flux can be calculated. Therefore, simplified cooling analytic solutions are implemented under COSI in addition to the irradiation surrogate models.

Besides, the list of isotopes tracked (321 isotopes with CESAR5.3) can be reduced in the COSI sped up version in order to further shorten the COSI calculation time. Both for irradiation and cooling calculations, output isotopes $j$ are chosen among whom mostly contribute to the fuel mass and post-processing results. The following isotopes constitute more than $99.999 \%$ of the spent fuel actinide mass after irradiation and thus are estimated:

$-{ }^{234} \mathrm{U},{ }^{235} \mathrm{U},{ }^{236} \mathrm{U},{ }^{238} \mathrm{U} ;$
$-{ }^{237} \mathrm{~Np},{ }^{239} \mathrm{~Np} ;$
$-{ }^{238} \mathrm{Pu},{ }^{239} \mathrm{Pu},{ }^{240} \mathrm{Pu},{ }^{241} \mathrm{Pu},{ }^{242} \mathrm{Pu} ;$
$-{ }^{241} \mathrm{Am},{ }^{242 \mathrm{~m}} \mathrm{Am},{ }^{243} \mathrm{Am} ;$
${ }^{242} \mathrm{Cm},{ }^{243} \mathrm{Cm},{ }^{244} \mathrm{Cm},{ }^{245} \mathrm{Cm},{ }^{246} \mathrm{Cm}$.

Several fission products such as ${ }^{90} \mathrm{Sr},{ }^{90} \mathrm{Y},{ }^{137} \mathrm{Cs}$ and ${ }^{137 \mathrm{~m}} \mathrm{Ba}$ complete the list to make possible estimating decay heat and radiotoxicity under long cooling period in waste. It is noteworthy that the choice of isotopes $j$ depends on the COSI outputs taken into account for optimization studies.

COSI sped up version is validated for a scenario of SFR deployment studied in this frame $[9,10]$. The nuclear power distribution of this scenario is represented in Figure 4.

First, all the actinide masses in cycle are compared from 2010 to 2140 . The results for the actinide elements are shown in Table 2.

Isotope estimation errors in cycle (waste excluded) are on the whole lower than $1.5 \%$ except $2.5 \%$ for ${ }^{243} \mathrm{Cm}$ estimation (present in low quantity). There is no transmutation in the application scenario so waste estimation errors are larger than cycle estimation errors: errors are lower than $3 \%$ except $4.5 \%$ for ${ }^{239} \mathrm{~Np}$ (present in low quantity), ${ }^{238} \mathrm{Pu}$ and ${ }^{240} \mathrm{Pu}$. Decay heat and radiotoxicity by ingestion for waste are calculated under long cooling period (from 1 to $10^{4}$ years after 2140), estimation

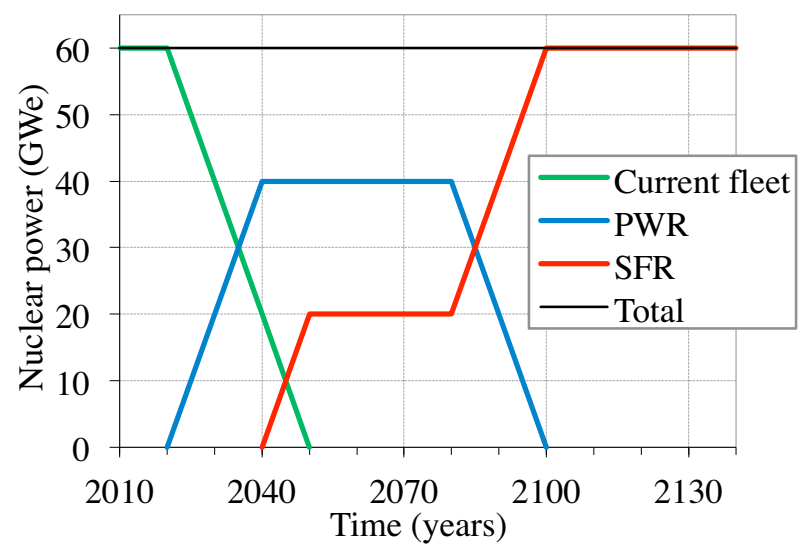

Fig. 4. Application scenario nuclear power distribution for validating surrogate models.

errors are no larger than $4 \%$. Finally, the number of High Level Waste (HLW) packages cumulated at the end of the scenario is estimated with an error of $1.2 \%$. These results are considered satisfactory enough to use COSI sped up version for optimization studies.

There are two types of COSI computation:

- standard: main depletion calculations at each date of interest (loading and unloading fuel dates, etc.);

- advanced: standard simulation plus additional depletion calculations; the advanced simulation considers the calculation of all the inventories in cycle for each year.

Computation time saving using COSI sped up version for the application scenario simulation is shown in Table 3 . It should be mentioned that COSI sped up version calculations are multi-threaded.

Table 2. Maximal relative errors for the actinide mass estimations with COSI sped up version for the application scenario simulation.

\begin{tabular}{lll}
\hline Element & $\begin{array}{l}\text { In cycle }(\%) \\
\text { (waste excluded) }\end{array}$ & In waste $(\%)$ \\
\hline $\mathrm{Pu}$ & 0.51 & 3.1 \\
$\mathrm{~Np}$ & 1.5 & 2.5 \\
$\mathrm{Am}$ & 0.95 & 2.8 \\
$\mathrm{Cm}$ & 0.68 & 2.0 \\
\hline
\end{tabular}


Table 3. COSI computation time decomposition for the application scenario simulation.

\begin{tabular}{lll}
\hline COSI version & Standard & Advanced \\
\hline COSI/CESAR5.3 & $4622 \mathrm{~s}$ & $46,791 \mathrm{~s}$ \\
Sped up & $38 \mathrm{~s}$ & $65 \mathrm{~s}$ \\
Speedup & $\times 122$ & $\times 720$ \\
\hline
\end{tabular}

An optimization calculation is then feasible using COSI sped up version because of the good surrogate models precision and the resulting time savings.

\section{Optimization exercise}

\subsection{Optimization problem definition}

Determining the best set of scenario parameters for a given problem requires that we define criteria, constraints and a base scenario with variables. In order to define this base scenario, it is necessary to make assumptions about the nuclear fleet evolution.

In the frame of a first application of the methodology, it is supposed that:

- SFR deployment is possible from 2040;

- all the reactors deployed from 2020 have a life span of 60 years;

- the nuclear fleet power equals to $60 \mathrm{GWe}$ from 2010 to 2140 to maintain a constant nuclear energy production;

- the current fleet phases out from 2020 to 2050 at the pace of $-2 \mathrm{GWe}$ year;

- there is no MOX fuel loaded in $\mathrm{EPR}^{\mathrm{TM}}$ from 2020, which is a simplification for the current study.

These assumptions have as consequences:

- the nuclear power distribution of the base scenario cannot be changed from 2010 to 2040; the current PWR fleet (UOX and MOX fuels) is partially renewed with EPR ${ }^{\mathrm{TM}}$ (only UOX fuel) from 2020 to 2040;

- the paces of reactors deployment and shutdown are respectively set to 2 and $-2 \mathrm{GWe} /$ year;

- there are two phases where reactors can be deployed from 2040: from 2040 to 2050 noted phase 1 and from 2080 to 2110 noted phase 2 .

We also consider that $\mathrm{EPR}^{\mathrm{TM}}$ are deployed before SFR in a same phase of reactors deployment. An example of this base scenario is shown in Figure 5 with respectively 7 and 27 SFR deployed during the phases 1 and 2 .

Two types of reactors can be deployed: $\mathrm{EPR}^{\mathrm{TM}}$ (UOX fuel) and SFR with their characteristics listed in Table 4. During the phase 1, 14 reactors need to be deployed to keep a nuclear power of $60 \mathrm{GWe}$. During the phase 2, 40 reactors have to be deployed to renew the nuclear fleet. Let $N_{1} \in[0,14]$ (resp. $N_{2} \in[0,40]$ ) be the number of SFR deployed during the phase 1 (resp. 2). The optimization study presented below only considers $N_{1}$ and $N_{2}$ as variables. Consequently, the scenarios are defined according

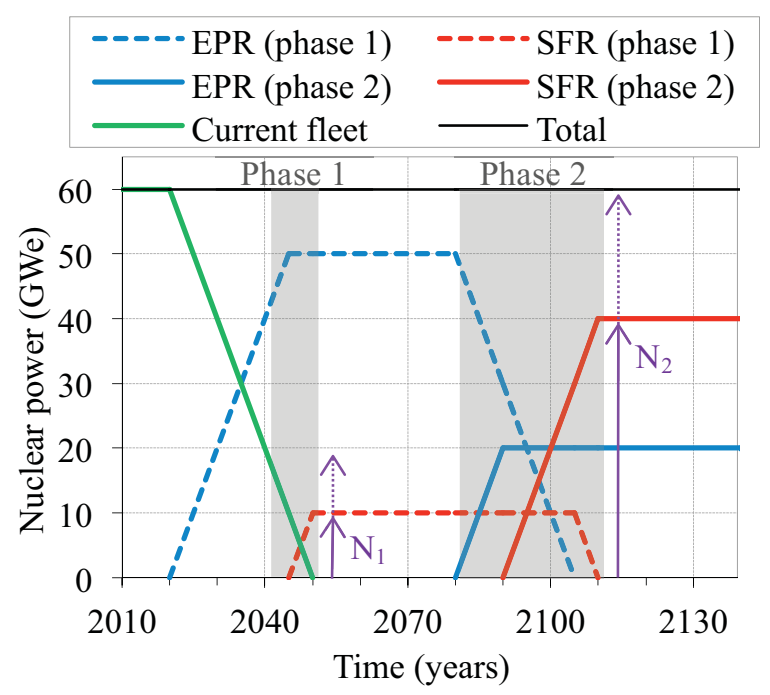

Fig. 5. Nuclear power distribution of the base scenario with the variables in purple (scenario noted $\{7,27\}$ ).

to the notation $\left\{N_{1}, N_{2}\right\}$. The scenario represented in Figure 4 corresponds to the case $\{14,40\}$.

The optimization problem aims to analyse the best SFR deployment scenarios. SFR deployment requires enough plutonium to ensure its fuel loadings are possible during its life span. Therefore, the lack of plutonium noted $\mathrm{m}_{\mathrm{Pu}}$ defined as the need of additional plutonium to make possible the scenario application needs to be zero. The reprocessing strategy is thus defined to ensure that all the spent fuels available can be reprocessed. In a first reprocessing strategy called Rep1, it is chosen that the SFR MOX fuel assemblies are reprocessed first when available, then the PWR (current fleet and $\mathrm{EPR}^{\mathrm{TM}}$ deployed before 2040) fuel assemblies. Rep1 aims to make the most of plutonium multirecycling in SFR fuels. A second strategy called Rep2 reverses the reprocessing order between PWR and SFR fuels. Rep2 aims to diminish the spent fuels accumulated. The annual reprocessing capacity is not limited in this study and is only regulated by fresh fuel fabrication needs. The two reprocessing strategies considered thereafter are reminded in Table 5. It is noteworthy that these assumptions on reprocessing are not representative of an industrial reality but avoid additional constraints on results for simplification purpose.

We consider two criteria in the optimization problem:

- the natural uranium mass consumption from 2010 to 2140 noted $\mathrm{m}_{\text {natU }}$ should be minimized; this criterion refers to safeguard natural resources;

Table 4. Base scenario reactors assumptions.

\begin{tabular}{lll}
\hline Reactors & $\mathrm{EPR}^{\mathrm{TM}}$ & $\mathrm{SFR}$ \\
\hline Electrical power & $1.5 \mathrm{GWe}$ & $1.5 \mathrm{GWe}$ \\
Net yield & $34.4 \%$ & $40.3 \%$ \\
Load factor & $81.8 \%$ & $81.8 \%$ \\
Core management & $4 \times 367 \mathrm{EFPD}$ & $5 \times 388 \mathrm{EFPD}$ \\
Average burnup & $55 \mathrm{GWd} / \mathrm{tHM}$ & $116 \mathrm{GWd} / \mathrm{tHM}$ \\
Fuel type & UOX $17 \times 17$ & MOX CFV-v1 [11] \\
\hline
\end{tabular}


Table 5. Base scenario possible reprocessing strategies.

\begin{tabular}{ll}
\hline Strategy & Reprocessing order of priority \\
\hline Rep1 & SFR MOX $\rightarrow$ PWR MOX $\rightarrow$ UOX $\rightarrow$ ERU \\
Rep2 & PWR MOX $\rightarrow$ UOX $\rightarrow$ ERU $\rightarrow$ SFR MOX \\
\hline
\end{tabular}

- the number of HLW vitrified packages produced from 2010 to 2140 noted $\mathrm{N}_{\mathrm{HLW}}$ should be minimized; this criterion refers to the reduction of nuclear waste production.

The production of HLW vitrified packages is determined according to the waste inventory so as to respect two conditions:

- the mass of fission products and actinides per package should be smaller than $70 \mathrm{~kg}$;

- the alpha radiation cumulated number over 10,000 years per gram of glass is limited to $2 \times 10^{19}$.

The HLW packages are produced after element separation during the spent fuel reprocessing. The reprocessing only occurs when SFR fresh fuel fabrication is required.

Thus the optimization problem is defined as follows:

$$
\begin{aligned}
& \min m_{\text {nat }}\left(N_{1}, N_{2}\right) \text { and } N_{H L W}\left(N_{1}, N_{2}\right) \\
& \text { with } N_{1}=0,1, \ldots, 14 \text { and } N_{2}=0,1, \ldots, 40 \\
& \text { such as } m_{P u-}\left(N_{1}, N_{2}\right)=0 t .
\end{aligned}
$$

The optimal scenarios for the combinatorial problem defined by equation (5) can be listed without using an optimization method as all the combinations can be simulated over a sensible time. It is necessary to compare the different scenarios to get the objective (resp. variable) trade-off surface named the Pareto front (resp. set), i.e. all the optimal scenarios in the objective (resp. variable) space. Optimal scenarios are defined as scenarios that cannot be improved in any of the criteria without degrading at least one of the other criteria. By definition, a scenario is said to dominate another one if all the criteria are improved or kept constant; at least one criterion has to be improved. A scenario which is not dominated by another one is optimal. Hence, we classify the scenarios into different designations:

- unfeasible scenarios (with $\mathrm{m}_{\mathrm{Pu}_{-}}>0 \mathrm{t}$ );

- feasible scenarios (with $\mathrm{m}_{\mathrm{Pu}_{-}}=0 \mathrm{t}$ );

- optimal scenarios (feasible and not dominated).

The results for the current problem are presented below.

\subsection{Results for the optimization problem using the first reprocessing strategy (Rep1)}

In this part, the objective functions and the Pareto set determined by comparing all the scenarios are analysed for the Rep1 strategy.

First we estimate $\mathrm{m}_{\mathrm{Pu}-}$ in order to define feasible scenarios for all the combinations $\left\{N_{1}, N_{2}\right\}$ (see Fig. 6). Unfeasible scenarios are those with a high number of

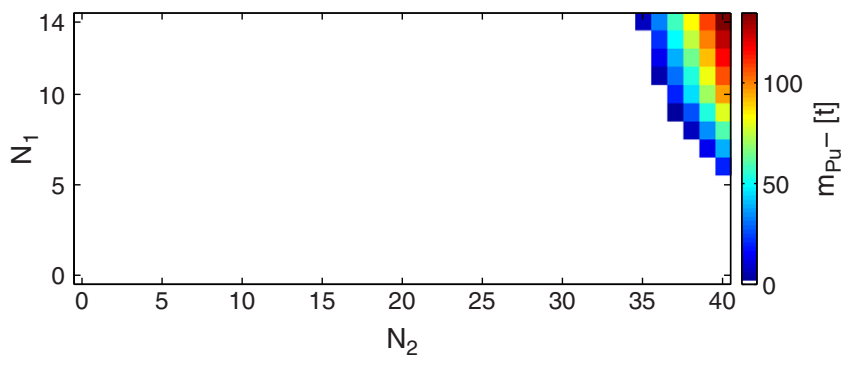

Fig. 6. Lack of plutonium for all $\left\{N_{1}, N_{2}\right\}$ (Rep1).

deployed SFR due to the increased need of plutonium to supply SFR. It is noteworthy that the application scenario $\{14,40\}$ does not respect the constraint because it is a simplified version of those studied in past works $[9,10]$.

Then we calculate the objective functions associated to all the combinations $\left\{N_{1}, N_{2}\right\}$ (see Fig. 7). The natural uranium consumption increases while the number of SFR deployed $N=N_{1}+N_{2}$ decreases as only EPR ${ }^{\mathrm{TM}}$ fuel holds natural uranium. The number of HLW vitrified packages increases while the number of SFR deployed increases as only SFR fuel fabrication needs to reprocess spent fuels. The isometric lines of the number of HLW packages do not follow $N$ mainly because of the reprocessing strategy. Indeed the quantity of reprocessed fuels depends on the fuel type. Besides it is noteworthy that $\mathrm{m}_{\text {natU }}$ and $\mathrm{N}_{\mathrm{HLW}}$ functions do not take into consideration the period after 2140 where phase $2 \mathrm{EPR}^{\mathrm{TM}}$ and SFR are shutdown.

Figure 8 represents the reprocessing flow distribution according to the spent fuel types and the HLW packages annual production for the scenario $\{14,34\}$. We can observe that the choice of reprocessing fuel type order greatly influences the HLW packages production as fuel types hold different plutonium content (see Tab. 6). Noteworthy that

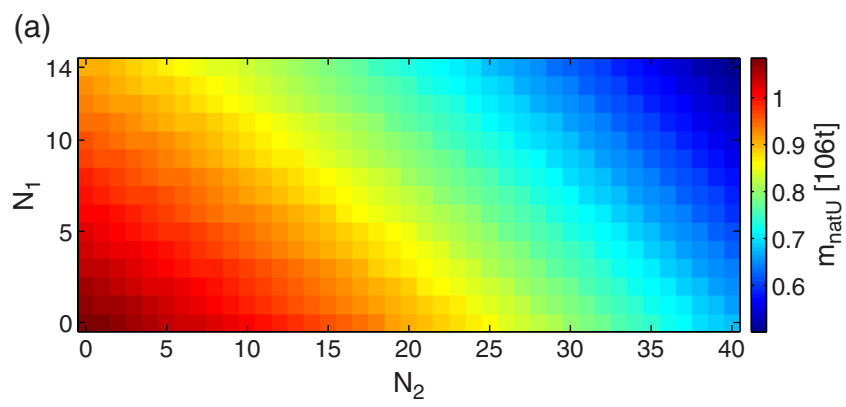

(b)

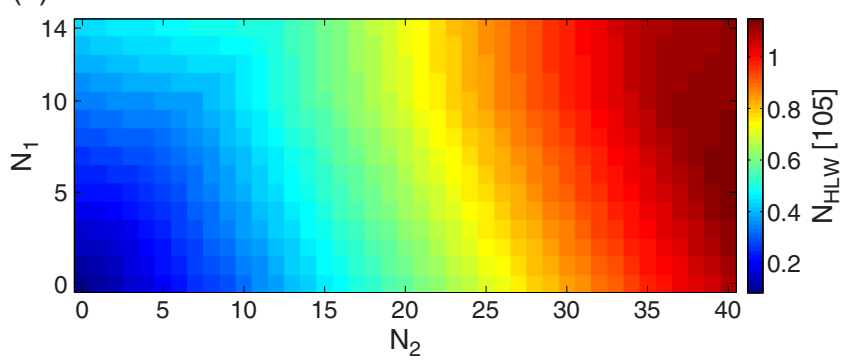

Fig. 7. Objective functions for all $\left\{N_{1}, N_{2}\right\}$ (Rep1). 


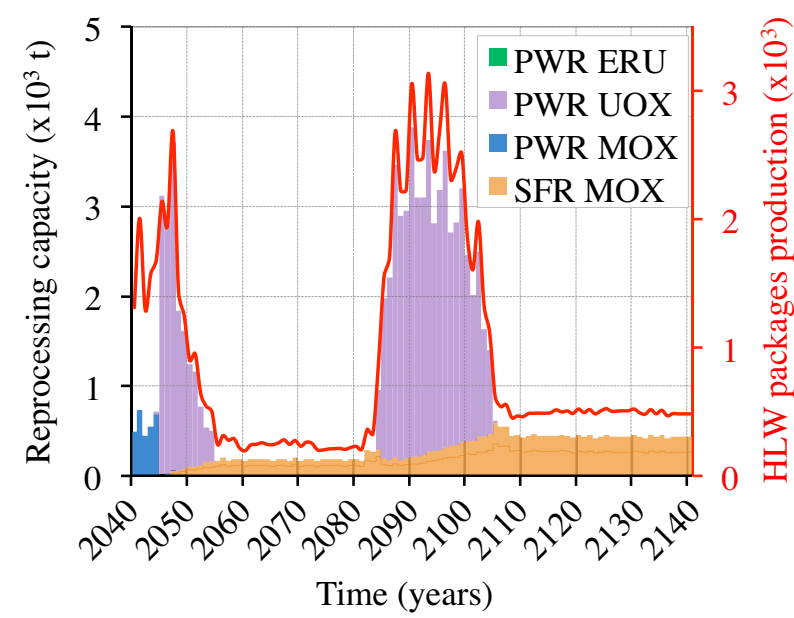

Fig. 8. Reprocessing flow and annual number of HLW packages produced for the scenario $\{14,34\}$ (Rep1).

Figure 8 suggests a significant fluctuation in reprocessing flow and thus costs implications. Indeed reprocessing capacity is one of the cost drivers for any closed fuel cycle. Stabilising the reprocessing capacity over long periods is not considered for the current optimization study but should be taken into consideration in further studies.

From all the 615 combinations, Figure 9 represents the unfeasible (34 combinations), feasible and optimal (66 combinations) scenarios for the optimization problem. The Pareto set (green coloured) shows that the optimal SFR deployment roughly consists in partly renewing during the phase 2 the SFR fleet deployed during the phase 1. If $N_{1}=14$, the scenarios with $10 \leq N_{2} \leq 34$ are optimal.

Figure 10 represents the scenarios in the objective space, with the Pareto front green coloured. It shows that increasing $\mathrm{m}_{\text {natU }}$ leads to decrease $\mathrm{N}_{\mathrm{HLW}}$ at the pace of about -1 HLW package for an additional consumption of natural uranium of 5 tons for the optimal scenarios. The choice of one optimal scenario among the Pareto set will depend on the preference on the criteria formulated by the decision maker.

\subsection{Results for the optimization problem using the second reprocessing strategy (Rep2)}

Now we consider the Rep2 strategy where the PWR fuels are reprocessed before the SFR fuels. The HLW packages production objective function (see Fig. 11) and optimal scenarios (see Figs. 12 and 13) for the optimization problem are represented below. The natural uranium consumption

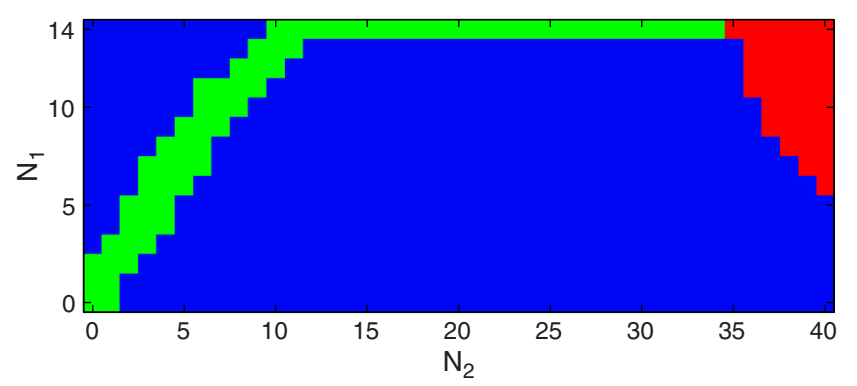

Fig. 9. Variable space: unfeasible, feasible and optimal scenarios are respectively red, blue and green coloured (Rep1).

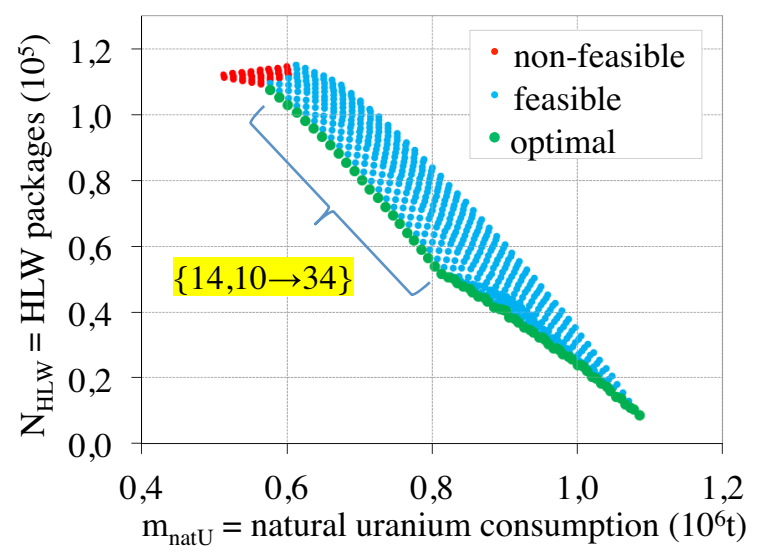

Fig. 10. Objective space: the Pareto front is green coloured (Rep1).

remains unchanged and the lack of plutonium is not significantly modified.

The change in reprocessing strategy results in a high modification of the number of HLW packages objective function. In fact, this strategy leads to a high HLW packages production while the first SFR are deployed then

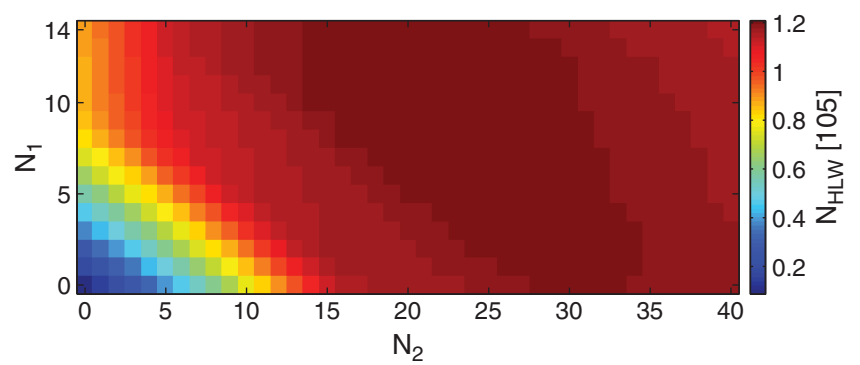

Fig. 11. HLW packages production objective function for all $\left\{N_{1}\right.$, $\left.N_{2}\right\}$ (Rep2).

Table 6. Number of HLW packages per ton of Pu extracted according to the fuel type reprocessed for the scenario $\{14,34\}$ (Rep1).

\begin{tabular}{llll}
\hline Fuel type & Reprocessing year & Pu content $(\%)$ & HLW packages $/$ ton of $\mathrm{Pu}$ \\
\hline PWR MOX & 2041 & 5.0 & 55 \\
PWR UOX & 2045 & 1.0 & 69 \\
SFR MOX & 2055 & 15 & 10 \\
\hline
\end{tabular}




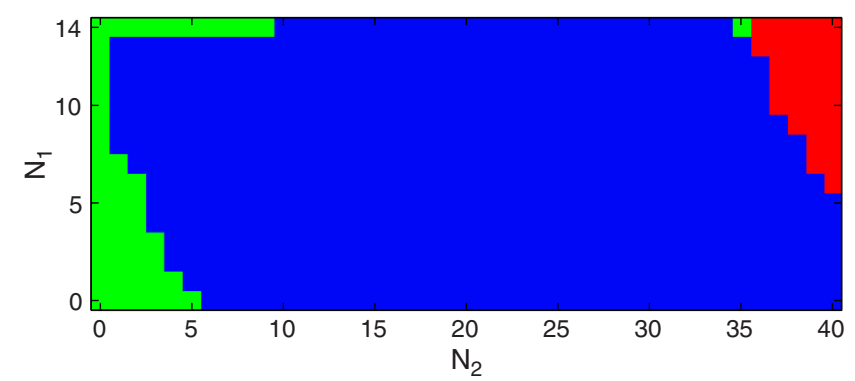

Fig. 12. Variable space: unfeasible, feasible and optimal scenarios are respectively red, blue and green coloured (Rep2).

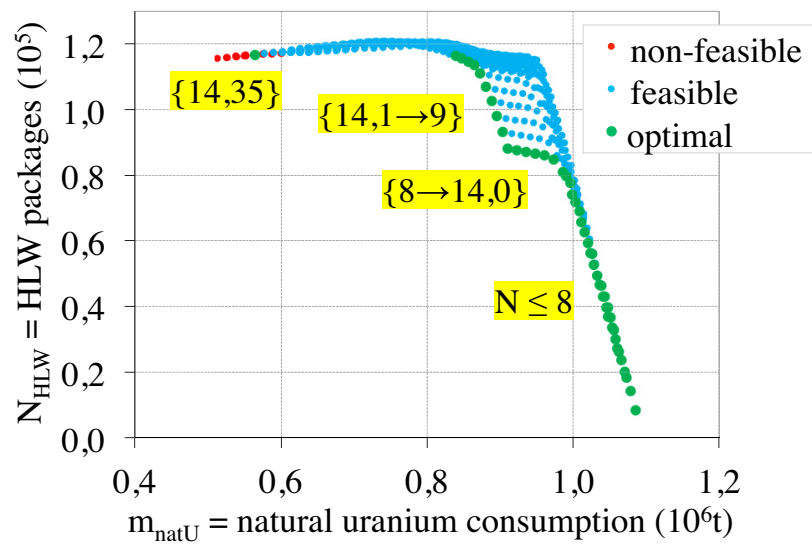

Fig. 13. Objective space: the Pareto front is green coloured (Rep2).

a lower production for the next ones (see Fig. 14). The HLW packages production slightly decreases from about $N_{2}=25$. Indeed increasing $N_{2}$ leads to an increase in the quantity of SFR fuels available for reprocessing and to a decrease in the quantity of PWR fuels. Table 6 shows that reprocessed PWR fuels to obtain a given amount of fissile materials leads to a higher number of HLW packages than SFR fuels.

The change in the number of HLW packages objective function leads to a different Pareto set (see Fig. 12). The

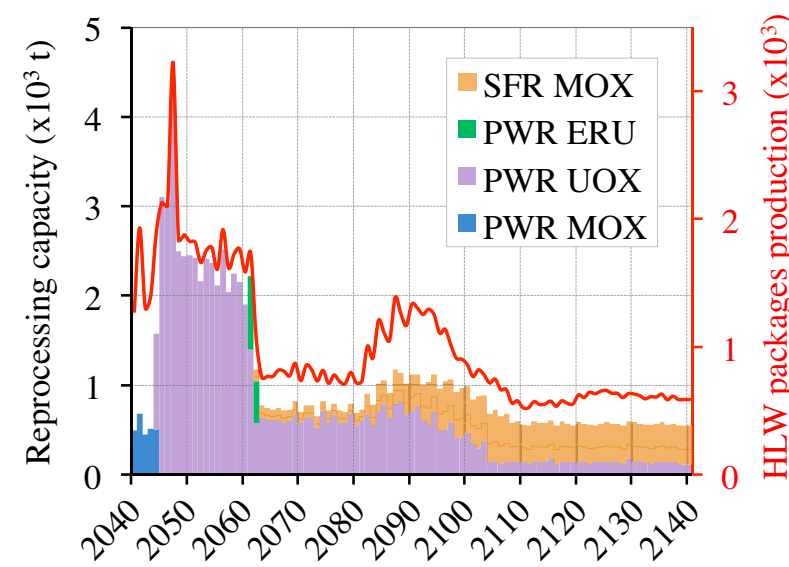

Time (years)

Fig. 14. Reprocessing flow and annual number of HLW packages produced for the scenario $\{14,34\}$ (Rep2).
Pareto set follows $N_{2}=0$ then $N_{1}=14$ until the scenario $\{14,9\}$ plus additional optimal scenarios for $N \leq 8$ plus the scenario $\{14,35\}$. There is no optimal scenario for $24 \leq N \leq 48$.

The Pareto front represented in Figure 13 is also greatly different, with a global deterioration (see Fig. 15) compared to the Pareto front with the Rep1 strategy.

This degradation results on the higher number of HLW packages produced with the Rep2 strategy. Besides some optimal scenarios have a slightly lower value on a criterion at the expense of a greatly higher value on the other one criterion. For example, the optimal scenarios $\{8$ to 14,0$\}$ have a gain much less pronounced on the number of HLW packages by increasing the natural uranium consumption than the other optimal scenarios with $N \leq 23$.

These results point out the need to consider reprocessing features as optimization variables (ongoing studies). These variables are related to the reprocessing order considering potential different spent fuel types mixing strategies.

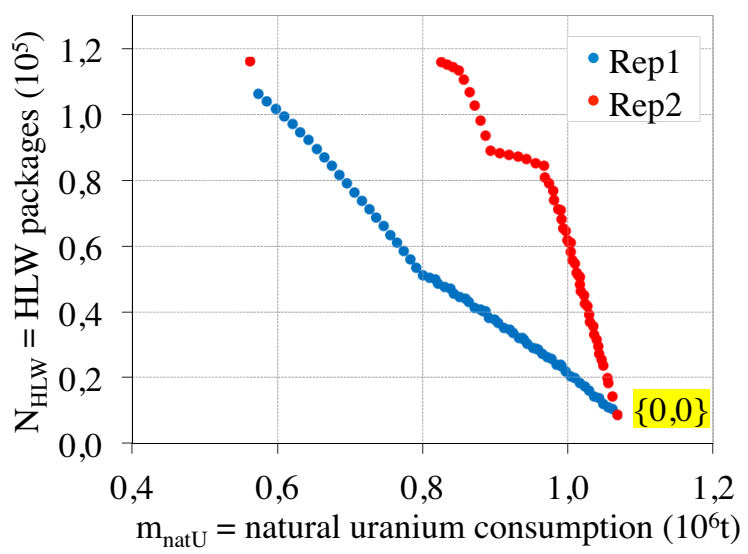

Fig. 15. Pareto fronts for Rep1 and Rep2 strategies.

(a)

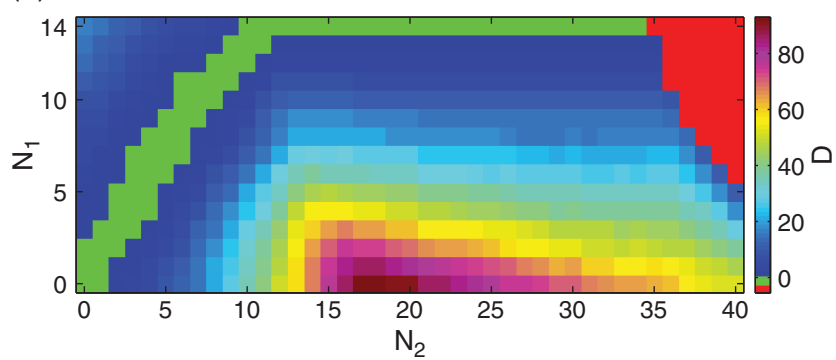

(b)

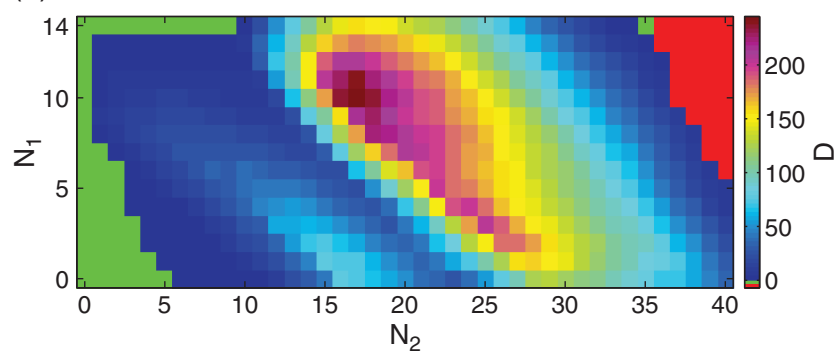

Fig. 16. Feasible solution depths for Rep1 (a) and Rep2 (b) strategies: unfeasible and optimal scenarios are respectively red and green coloured. 
(a)

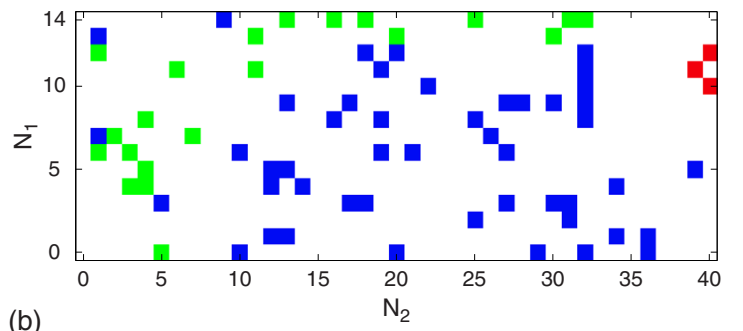

(b)

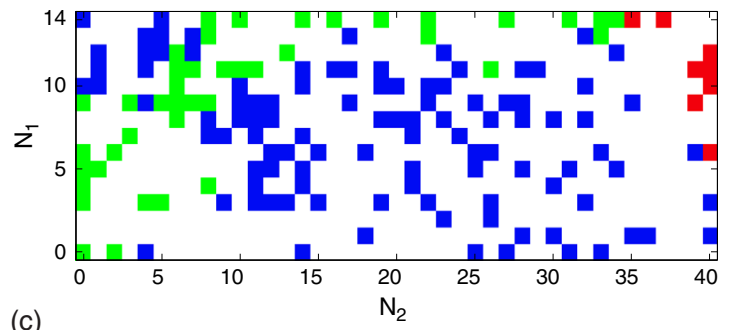

(c)

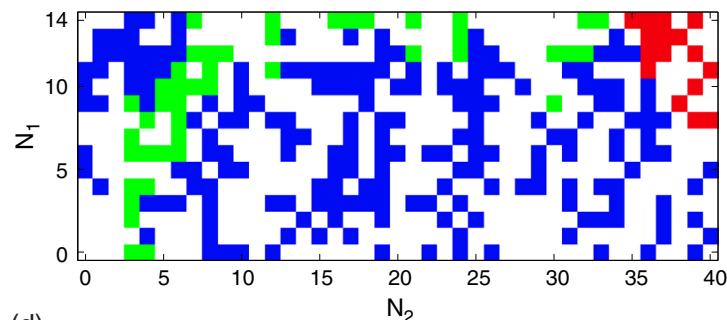

(d)

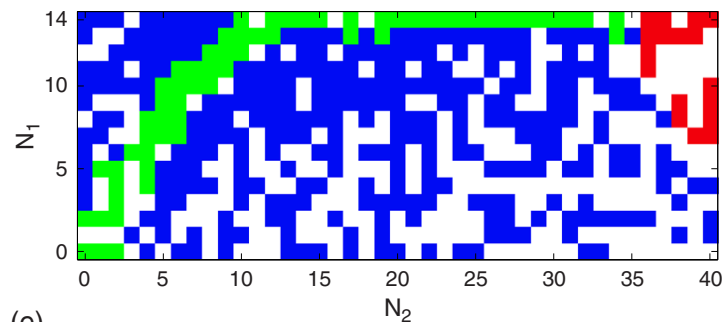

(e)

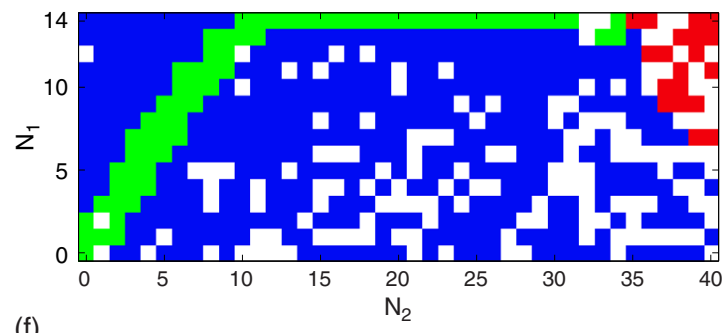

(f)

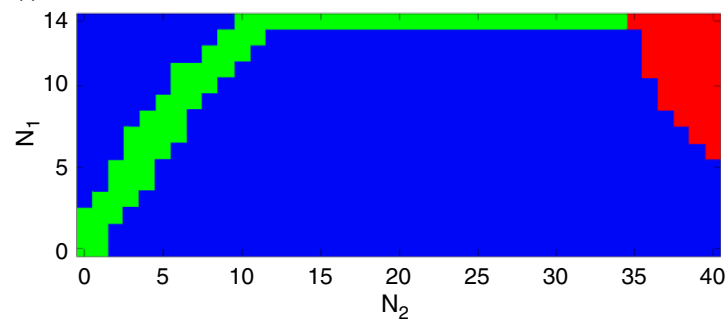

Fig. 17. Variable space for different GA population sizes $(30,50$, 100, 200 and 300): non-evaluated, unfeasible, feasible and optimal scenarios are respectively white, red, blue and green coloured (Rep1); Figure 9 is reminded in (f). (a)

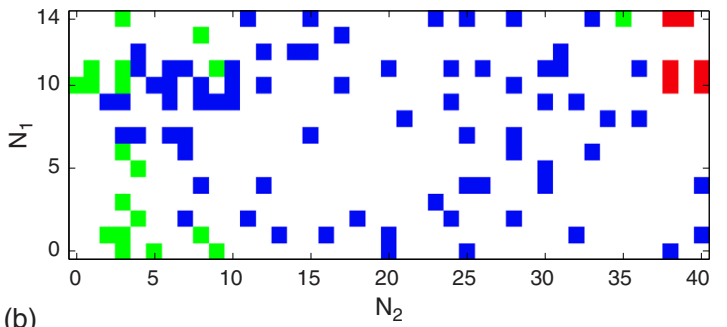

(b)

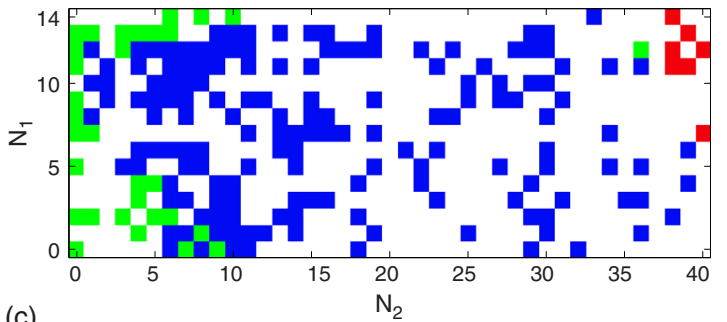

(c)

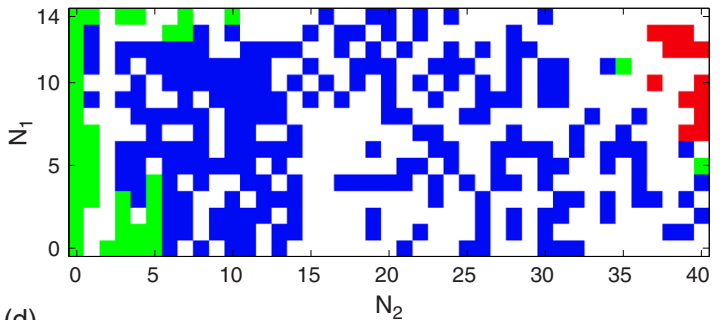

(d)

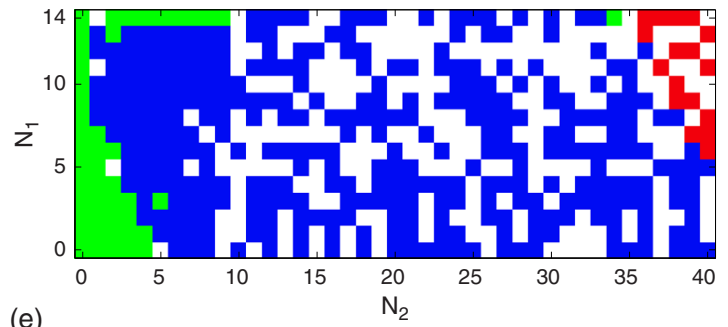

(e)

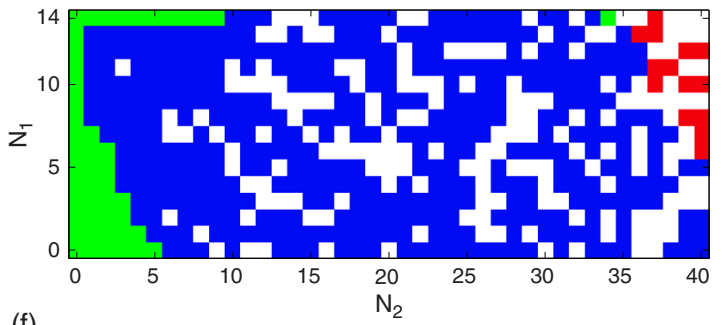

(f)

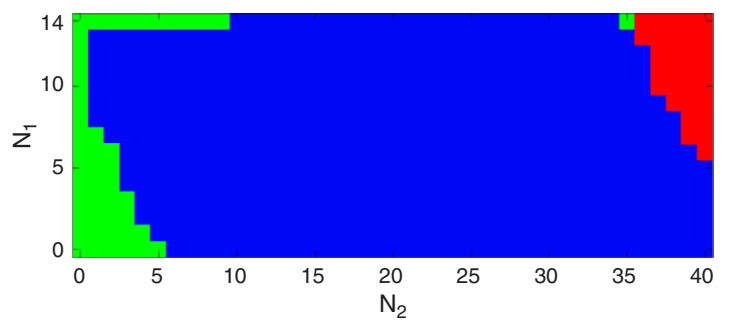

Fig. 18. Variable space for different GA population sizes $(30,50$, 100, 200 and 300): non-evaluated, unfeasible, feasible and optimal scenarios are respectively white, red, blue and green coloured (Rep2); Figure 12 is reminded in (f). 


\subsection{Results using a genetic algorithm method}

Different stochastic optimization methods can be used to solve an optimization problem. A genetic algorithm (GA) method available on the URANIE platform is chosen. The GA method considers parameters which define the balance between scenarios exploration (filling the space not to converge towards local optimal solutions) and exploitation (reducing the search space to converge towards optimal solutions). The aim of this example is to illustrate and test the functioning of the methodology for an easy problem. Otherwise using an optimization method is unnecessary to solve the considered problem because all the combinations can be estimated over a sensible time.

We define the depth noted $D$ of a feasible scenario as the number of scenarios which dominate this scenario. Figure 16 shows that the scenarios close to the Pareto set have a low depth and may be dominated by only one optimal scenario. Therefore, all the optimal scenarios should be reached to avoid the low-depth scenarios being considered as optimal by the method. These figures also confirm that the optimization method needs to have a good balance between exploring the variable space to reach the dark blue coloured subspace and exploiting this subspace to reach the green coloured Pareto set.

The population size is one of the GA parameters which has an impact on the number of evaluated scenarios during the optimization process. Figures 17 and 18 show the solutions evaluated by the GA method for different population sizes with the two reprocessing strategies. These figures show that the GA method leads to the Pareto set but needs to evaluate a high number of different scenarios.

The advantage of using an optimization method such as the GA method is yet to be tested in further more realistic optimization studies (addition of variables and objectives) where all the feasible solutions cannot be simulated. Ongoing studies consider continuous optimization problem with a much higher number of variables and then might require changing the GA parameters to converge on a good quality Pareto continuous set.

\section{Conclusions}

The consequences of strategic choices on material inventories and flux in the fuel cycle can be analysed with COSI. Indeed COSI enables to compare various fleet evolution options (e.g. new reactor systems deployment) and different nuclear material managements (e.g. plutonium multi-recycling). COSI is coupled with the CESAR depletion code.

In this paper, a methodology for the nuclear fleet evolution scenarios optimization using COSI is introduced. A large number of scenario calculations is needed to solve an optimization problem, which makes infeasible an optimization calculation using COSI. Given that CESAR calculations represent about $95 \%$ of the COSI computation time, CESAR irradiation surrogate models carrying out with ANN regression method and cooling analytic models have been coupled with COSI. The outputs of interest estimated by the COSI sped up version using these simplified models have an estimation error of about $1 \%$ for the cycle (waste excluded) actinide masses, $3 \%$ for the waste and $1.2 \%$ for the number of HLW packages produced. These results are considered satisfactory for optimization studies. The time saving using the COSI sped up version can vary from about 120 to 720 according to the COSI calculation type. This time saving makes feasible an optimization calculation over a sensible time.

An example of optimization study is presented using a base scenario inspired by the studies done in the frame of the 2006 French Act for waste management. The optimization problem involves two discrete variables related to the number of deployed SFR to renew the French PWR fleet and two criteria: minimizing the natural uranium consumption and the number of produced HLW vitrified packages. The Pareto set of this combinatorial problem can be exactly calculated to validate the optimization results using a genetic algorithm method. The main conclusion is that further studies need considering reprocessing features (order of priority and quantity of reprocessed fuel type) as optimization variables to make the problem more realistic. The advantage of using an optimization method such as the GA method is yet to be tested in further continuous studies where all the feasible solutions cannot be simulated. Besides the list of criteria should be completed by economic and safety considerations. It is noteworthy that obtaining a single optimal scenario from the Pareto set requires formulating preferences on the criteria, which depends on the decision maker.

\section{Nomenclature}

ANN Artificial Neural Network

CESAR depletion code

COSI scenarios simulation code

EFPD Effective Full-Power Day

$\mathrm{EPR}^{\mathrm{TM}}$ European Pressurized Reactor (EPR is a trademark of the AREVA group)

ERU re-Enriched Reprocessed Uranium

GA Genetic Algorithm

GP Gaussian Process

HLW High Level Waste

LHS Latin Hypercube Sampling

LR Linear Regression

MOX Mixed OXide

PR second-order Polynomial Regression

PWR Pressurized Water Reactor

SFR Sodium-cooled Fast Reactor

tHM ton of Heavy Metal

UOX Uranium OXide

\section{References}

1. C. Coquelet-Pascal et al., COSI6: a tool for nuclear transition scenario studies and application to SFR deployment scenarios with minor actinide transmutation, Nucl. Technol. 192, 91 (2015) 
2. J.-M. Vidal et al., CESAR5.3: an industrial tool for nuclear fuel and waste characterization with associated qualification, in Waste Management 2012, Phoenix, USA (2012)

3. F. Gaudier, URANIE: the CEA/DEN uncertainty and sensitivity platform, Proc. Soc. Behav. Sci. 2, 7660 (2010)

4. R. Hays, P. Turinsky, Stochastic optimization for nuclear facility deployment scenarios using VISION, Nucl. Technol. 186, 76 (2014)

5. S. Passerini et al., A systematic approach to nuclear fuel cycle analysis and optimization, Nucl. Sci. Eng. 178, 186 (2014)

6. G. Krivtchik et al., Development of depletion code surrogate models for uncertainty propagation in scenarios studies, in SNA + MC 2013, Paris, France (2013)

7. G. Krivtchik et al., Analysis of uncertainty propagation in scenario studies: surrogate models application to the
French historical PWR fleet, in GLOBAL 2015, Paris, France (2015)

8. B. Iooss et al., Numerical studies of the metamodel fitting and validation processes, Int. J. Adv. Syst. Meas. 3, 11 (2010)

9. C. Coquelet et al., Comparison of different options for transmutation scenarios studied in the frame of the French law for waste management, in GLOBAL 2009, Paris, France (2009)

10. C. Coquelet-Pascal et al., Comparison of different scenarios for the deployment of fast reactors in France - Results obtained with COSI, in GLOBAL 2011, Makuhari, Japan (2011)

11. B. Fontaine et al., The French R\&D on SFR core design and ASTRID Project, in GLOBAL 2011, Makuhari, Japan (2011)

Cite this article as: David Freynet, Christine Coquelet-Pascal, Romain Eschbach, Guillaume Krivtchik, Elsa Merle-Lucotte, Multiobjective optimization for nuclear fleet evolution scenarios using COSI, EPJ Nuclear Sci. Technol. 2, 9 (2016) 